
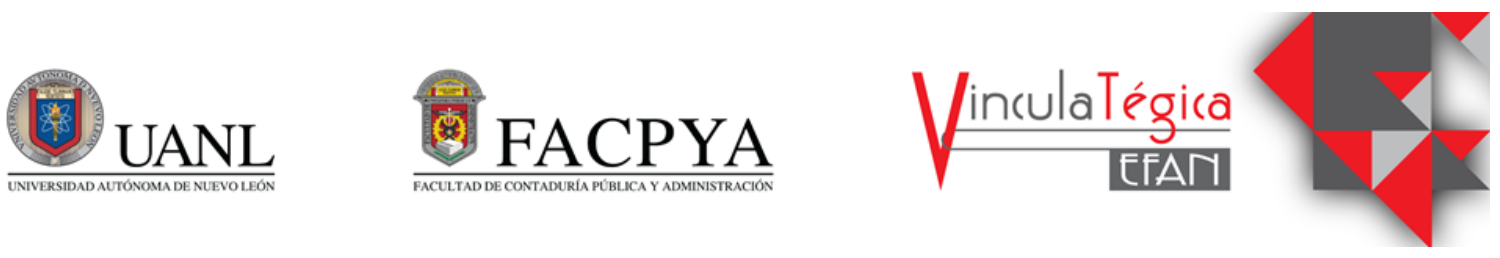

\title{
El uso y apropiación de las TIC's en las actividades académicas universitarias en la nueva modalidad en línea
}

\author{
Julio César Macías Villarreal ${ }^{1}$, José Rafael Baca Pumarejo ${ }^{2}$ y José Federico Delgado Garza ${ }^{3}$ \\ ${ }^{1}$ Universidad Autónoma de Tamaulipas, Facultad de Comercio y Administración Victoria, Tamaulipas, \\ México,jcmacias@docentes.uat.edu.mx, Centro Universitario S/N \\ C.P. 87149, (834) 3181800 \\ ${ }^{2}$ Universidad Autónoma de Tamaulipas, Facultad de Comercio y Administración Victoria, Tamaulipas, \\ México,rbaca@docentes.uat.edu.mx, Centro Universitario S/N \\ C.P. $87149,(834) 3181800$ \\ ${ }^{3}$ Universidad Autónoma de Tamaulipas, Facultad de Comercio y Administración Victoria, Tamaulipas, \\ México,fdelgado@docentes.uat.edu.mx, Centro Universitario S/N
} C.P. $87149,(834) 3181800$

\footnotetext{
Información del artículo revisado por pares

Fecha de aceptación: junio-2021

Fecha de publicación en línea: diciembre-2021

DOI: https://doi.org/10.29105/vtga7.1-158
}

\section{Resumen}

El uso de las tecnologías de la información y comunicación dentro de los procesos educativos universitarios es un tema de gran relevancia, ya que éstas brindan herramientas para apoyar a las actividades de enseñanza aprendizaje y con las cuales se puede mejorar su rendimiento académico, sin embargo su uso y apropiación enfrenta toda una serie de retos y desafíos. Esta investigación tiene como objetivo analizar la percepción del alumnado universitario respecto del uso y apropiación de las Tecnologías de la Información y Comunicación respecto de sus actividades docentes en la nueva modalidad en línea. Para esta investigación se ha tomado como caso la Facultad de Comercio y Administración Victoria dependiente de la Universidad Autónoma de Tamaulipas, así como una muestra tomada del periodo de agosto-diciembre del año 2020, siendo la población de 1664 estudiantes inscritos de las distintas carreras. Los resultados encontrados se direccionan a que el profesor y el alumno universitarios tendrán grandes retos que deberán afrontar, ya que se enfrentan a un contexto cambiante, por lo que deberán incorporarse a un proceso de adaptación continuo, y cada uno cumplir el rol que le corresponde, con el
Abstract

The use of information and communication technologies within university educational processes is an issue of great relevance, since they provide tools to support teaching-learning activities and with which their academic performance can be improved, however their use and appropriation faces a whole series of challenges and challenges. This research aims to analyze the perception of university students regarding the use and appropriation of Information and Communication Technologies regarding their teaching activities in the new online modality. For this research, the Victoria Faculty of Commerce and Administration, dependent on the Autonomous University of Tamaulipas, has been taken as a case, as well as a sample taken from the August-December period of 2020, with the population of 1664 students enrolled in the different careers. The results found indicate that the university professor and student will have great challenges that they will have to face, since they face a changing context, so they will have to join a continuous adaptation process, 
sentido de lograr alcanzar el proceso enseñanza aprendizaje en la nueva modalidad en línea.

Palabras clave: Actividades docentes, Modalidad en línea, Tecnologías de la información y comunicación, Universitarios.

\section{INTRODUCCIÓN}

La emergencia sanitaria originada por el COVID-19, ha provocado que de un día para otro, el alumnado universitario y sus docentes se hayan visto forzados a cambiar las aulas de clase por las habitaciones en sus casas, y transferir los contenidos de aprendizaje, las tareas y actividades a una modalidad en línea, a través de las Tecnologías de la Información y la Comunicación (TIC) en sus variadas formas: aplicaciones, páginas web, correo electrónico, videoconferencias, etc. (García, Rivero, \& Ricis, 2020).

El cierre de los espacios educativos, como medida para contener la pandemia establecido por las autoridades, han llevado a un acelerado replanteamiento del proceso educativo por parte de las instituciones educativas, brindado soluciones a través de la modalidad en línea para asegurar la continuidad académica. Las dificultades son variadas, desde la falta de conectividad, la carencia en equipos tecnológicos por parte de los alumnos y la adecuación de los programas de estudio, hasta profesores no capacitados para esta nueva normalidad.

La utilidad de las TIC en esta época son un componente indispensable en los procesos de interconectividad entre los individuos (Coll, 2004). Ubicándose como un componente fundamental de las mejoras y progresos en el contexto académico que existe en la actualidad, en específico con la educación superior, que cada vez ha cobrado suma importancia por lo que aporta al desarrollo socioeconómico.

Las TIC han favorecido a una labor colaborativa e interdisciplinaria en Licenciaturas como Contaduría Pública y Administración, por nombrar algunas, dando la posibilidad para que los universitarios incorporen innovadores recursos de tipo tecnológico y contribuyan en la interrelación and each one fulfills the role that corresponds to him. , in order to achieve the teaching-learning process in the new online mode.

Keywords: Teaching activities, Online modality, Information and communication technologies, University.

entre alumnos y el conocimiento (Caicedo y Rojas, 2014). Disponiendo en estos tiempos un cúmulo de recursos TIC adaptados a la docencia, con el único propósito de apoyar a los profesores en su impartición de catedra, y promover la utilización de tecnología en el alumnado; beneficiando así al desarrollo y progreso del sector educativo (Del Moral, Villalustre, \& Neira, 2014).

La educación universitaria tiende a la implementación de las TIC en sus procesos educativos, originado por el fácil acceso de los estudiantes a la información y a la tecnología, en un ambiente donde las recursos tecnológicos y los medios digitales se posicionan de manera relevante $y$ significativa, reconociendo la gran relevancia que originan las TIC en el avance de la sociedad (Sánchez, Pardo, \& Izquierdo, 2010).

El uso generalizado de las TIC, en el contexto universitario, se ha transformado en un apoyo didáctico innovador para el profesorado, y por otra parte el alumnado le permite estar en contacto con nuevos conocimientos, actitudes y valores a partir de un acceso sencillo a las fuentes de información, ayudando así al desarrollo de innovadoras habilidades tecnológicas y de comunicación que aporten en la medida de lo posible a la generación y transmisión de conocimientos (Gómez, 2008).

Los procesos de enseñanza aprendizaje se ven fortalecidos por los instrumentos y herramientas de las nuevas tecnologías, propiciando el mejoramiento continuo dentro de los campus universitarios, así como también contribuyendo a la interacción entre la comunidad, que involucra a profesores, alumnos, directivos y padres de familia. Nuestro campo laboral requiere profesionales formados para enfrentar las exigencias de un entorno globalizado, siendo el utilización de 
las TIC un vínculo para contribuir a la construcción de una sociedad más equitativa, pero todo estará supeditado de cómo se utilicen los conocimientos, así como los valores y los juicios críticos (Gómez \& Macedo, 2010).

El sector educativo se ve influenciado significativamente por las TIC, impactando en todos los grados, desde educación básica hasta el nivel superior. Evolucionamos a una era de innovadoras tecnologías utilizadas para el mejoramiento del proceso educativo, lo que lleva a la sociedad a realizar esfuerzos y trasladarse a una etapa de adaptación con la finalidad de adquirir conocimientos.

\section{MARCO TEÓRICO}

\subsection{Las TIC en el proceso de enseñanza aprendizaje}

La utilización de las tecnologías de la información y comunicación (TIC) en la enseñanza plantea grandes desafíos como herramienta para ser usada por los profesores, porque demandan transformaciones considerables en algunos aspectos. Una de estas modificaciones importantes es en el proceso educativo al apoyarse en las TIC y este cambio radica principalmente en tener el rol que fungirá el docente, ya que dejara de fungir como agente de acceso a la información y pasara a ser solamente una figura de acompañamiento para los estudiantes, facilitando y administrando el uso de las herramientas y medios de enseñanza, propiciando con esto el autoaprendizaje de los estudiantes guiado en todo momento por el maestro.

La utilización de las tecnologías se está integrando rápidamente en la vida cotidiana ya sea para realizar gestiones administrativas, tener accesibilidad a la información o puramente para la recreación y el entretenimiento, de acuerdo con Sáez (2010) este problema tendrá relevancia gradualmente en un entorno a futuro ya que los jóvenes y niños que se forman actualmente están más acostumbrados a interactuar de manera habitual con las herramientas tecnológicas.

Esto presume la necesidad de preparar y plantear un proceso educativo que aparte de desarrollar conocimientos generales y específicos, desarrolle las competencias respecto al uso de las TIC que son y serán demandadas tanto en el ambiente académico como en el profesional.

De acuerdo al escrito titulado "Nuevas tecnologías de la información y educación de adultos" realizado por la UNESCO (1997): "Las tecnologías de la información y comunicación son un instrumento poderoso que incrementa el poder de acceso a todo ciudadano y ciudadana a la información y a los nuevos métodos de educación, enriqueciendo su entorno de aprendizaje".

Las TIC han sido contempladas como opciones que permiten enseñar contenidos en forma pronta y generalizada además de hacer la posible conexión entre estudiantes y profesores ubicados en lugares distantes. Cuban (2001) sostiene que los métodos acostumbrados en la práctica docente, como depender de la bibliografía, instrucciones generalizadas, conferencias y exámenes de opción múltiple, están en obsolescencia en esta era de la información.

\subsection{La formación docente y las TIC en el proceso de educativo}

En esta nueva era un profesor que no utilice las TIC lo podemos considerar una deficiencia en relación con sus estudiantes, por lo que la inclusión de estas a la capacitación del profesorado es de suma relevancia, tanto para su propia preparación como para la enseñanza que puede ofrecerles. La capacitación y formación del maestro resulta una tarea esencial, pues una de las dificultades más frecuentes a las que se enfrentan los profesores se refiere al aspecto generacional. Según Kiridis, Drossos y Tsakiridou (2006), algunos maestros se les facilita adaptarse a estos cambios y hacer la transición a un entorno completamente nuevo de aprendizaje sin ningún problema, pero algunos pueden tener una forma de ser más conservadora que los orille a ver los cambios con mayor dificultad y les resulte complejo enfrentar ese paradigma que los obligue a modificar su forma de enseñar y compartir los conocimientos.

Cabero \& Llorente (2006), precisan que las nuevas tecnologías debe incorporarse en todo plan de capacitación del profesorado, de 
manera que los académicos universitarios tengan las competencias necesarias en TIC y les permita a través de estas desarrollar nuevas herramientas de enseñanza y de comunicación con los estudiantes permitiéndole a estos que sea más dosificada y de interés, facilitando con ello que adquieran mayor motivación y compromiso con la clase.

Ferro, Martínez y Otero (2009) señalan que el uso de las TIC en el proceso de enseñanza aprendizaje motiva al estudiantado y cautiva su atención, transformándose así en uno de los generadores de enseñanza, pero para poderlo realizar, es indispensable tener en cuenta la alfabetización digital que tanto maestros como estudiantes deben apropiarse, por lo que se considera importante elaborar un diagnóstico que permita conocer el nivel de apropiación digital que tienen los alumnos con el manejo de las tecnologías antes de implementar un proceso de enseñanza a través de la utilización de las TIC.

\subsection{Los estudiantes y las TIC en el proceso de enseñanza aprendizaje}

Actualmente, la metodología convencional ha consistido en recolectar la mayor cantidad de conocimientos posible, pero en un entorno en constante cambio esto no es conveniente, al estar con la incertidumbre de no saber si lo que se está estudiando será importante. Para los alumnos es indiscutible que se han beneficiado con el uso de las TIC, ya que su trabajo y tareas se han simplificado tanto para la búsqueda de información como la comunicación con el docente y sus compañeros.

Es importante considerar que al momento de implantar acciones educativas utilizando la tecnología se deben considerar algunas acciones relacionadas con la disponibilidad de herramientas tecnológicas, de infraestructura y de capacidad con las que cuentan los alumnos para generar actividades académicas vinculadas con la utilización, selección y organización de la información, de manera que el estudiante vaya integrándose como un futuro profesional de la sociedad de la información.

Para Mason (1998), el uso de las TIC

en la enseñanza da paso a nuevas oportunidades en relación a una educación de calidad, sostenida en medios a distancia cuyas estrategias son prácticas, ejercicios y casos tradicionales en la educación en las aulas, pero que ahora son sencillamente adecuadas en su formato virtual. Por otro lado, el conocer el tipo de tecnología con el que cuentan los alumnos será de gran relevancia para permitir el proceso de enseñanza aprendizaje, la cual debe de permitir la distribución de los contenidos, el acceso a la información, la interacción entre maestros y alumnos, la gestión del curso, la capacidad de control de los usuarios durante el desarrollo del curso, etc.

En conclusión, diseñar un entorno de formación adecuado a las capacidades de los alumnos supone participar de un conjunto de decisiones que permitan la utilización de la tecnología desde una perspectiva de formación flexible, adecuando un modelo pedagógico en el que se tenga interacción entre el alumno y el maestro. (Salinas, 1997) (Salinas, 1999) (Morán \& Myringer, 1999).

De acuerdo con lo establecido en distintos investigaciones, uno de los elementos medulares en la educación en línea es la conectividad entre el alumno y el maestro, en la que no solo es importante la cantidad (Castaño-Muñoz, Duart \& Vinuesa, 2014) sino también la calidad de la interacción (Brodie, Ilic, Juric, \& Hollebeek, 2013). La resultados en la práctica señala las ventajas de la enseñanza a través de medios sincrónicos en el sentido de que facilita más la proximidad que los asincrónicos (Baker, 2011; Chakraborty \& Nafukho, 2015) y corta la impresión de encierro al crear un efecto de pertenencia (Ragusa, 2017), además de mejorar el rendimiento. Sin embargo, existen autores que afirman en sus publicaciones las preferencias de los alumnos por un modelo de comunicación que combine ambas modalidades tanto sincrónicas como asincrónicas porque perfeccionan la práctica del aprendizaje (Moallen, 2015).

\section{MÉTODO}

La presente investigación podemos definir que es una investigación de tipo descriptiva, y se utiliza el método cuantitativo ya que se procedió a recolectar información, el diseño 
es no experimental ya que se basó en la aplicación de 321 cuestionarios a estudiantes inscritos de los programas de estudio de Contaduría Pública, Licenciatura en Administración, y de Licenciatura en Tecnologías de la Información de la Facultad de Comercio y Administración Victoria incorporada a la Universidad Autónoma de Tamaulipas en el periodo escolar agostodiciembre de 2020. Así mismo es una investigación documental, debido a que se utilizaron revistas, internet y bases de datos, además nos apoyamos en artículos de revistas y libros, así como de teorías aplicadas y las distintas aportaciones de diversos autores que nos brindaron información que nos ayudó a fundamentar la presente investigación.

\subsection{Población y Muestreo}

La Institución objeto de estudio fue la Facultad de Comercio y Administración Victoria incorporada a la Universidad Autónoma de Tamaulipas en el periodo escolar agosto-diciembre de 2020, siendo la población de 1664 estudiantes inscritos de los programas de estudio de Contaduría Pública, Licenciatura en Administración, y de Licenciatura en Tecnologías de la Información.

Para el cálculo de la muestra se utiliza la siguiente fórmula:

Donde:

$$
n=\frac{N * Z_{a}^{2} p * q}{d^{2} *(N-1)+Z_{a}^{2} p * q}
$$

N= Total población (1664 alumnos)

$\mathrm{n}=$ Tamaño de la muestra

$\mathrm{Za}=1.96$ al cuadrado (si la seguridad es de $95 \%)$

\section{RESULTADOS}

Los resultados muestran una participación de 321 registros de los cuales se ha resaltado la relevancia de que el alumnado y el profesorado universitario cuenten con las $\mathrm{p}=$ Proporción esperada. Se utiliza el valor $\mathrm{p}=$ $0.5(50 \%)$ que maximiza el tamaño de la muestra.

$\mathrm{q}=1-\mathrm{p}(1-0.5=0.5)$

$\mathrm{d}=$ Precisión (en la investigación se usará el $5 \%$ )

Resultado:

$n=\frac{1664 *\left(1.96^{2}\right) 0.5 * 0.5}{0.05^{2} *(1664-1)+1.96^{2} * 0.5 * 0.5}$ $\mathrm{n}=313$

En este estudio se tuvo la participación de 321 alumnos, con lo que cubre con el valor mínimo de la muestra calculada.

\subsection{Instrumentos de recolección de información}

Para alcanzar los objetivos propuestos para este trabajo de investigación, se adaptó el instrumento que fue elaborado por Sanabria y Hernández (2011), los cuales se apoyaron en estudios e investigaciones de científicos expertos en el tema, dicho instrumento estaba integrado por preguntas que cuentan con escalas tipo Likert de cinco puntos, que van desde "1 - Muy en desacuerdo" a "5 Muy de acuerdo". Realizándose adaptaciones para su aplicación, por lo que se tomaron algunas preguntas debido a su importancia para la línea de investigación en este estudio.

Por lo tanto, se cumple fielmente con la norma de Validez de Contenido del instrumento, en donde se establece la recomendación sobre el análisis del instrumento de recolección por tres o cinco expertos aproximadamente.

competencias necesarias en el uso y manejo de las Tecnologías de la Información y Comunicación para poder aprovecharlas de forma eficiente y ética en sus actividades de enseñanza-aprendizaje. 
Figura 1. Género

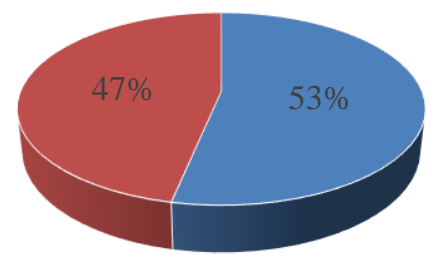

- Femenino

- Masculino

Fuente: elaboración propia.

La figura 1 muestra el porcentaje según el género del alumnado universitario que contribuyo en este trabajo de investigación, siendo el género femenino una mayoría en un
$53 \%$ y el género masculino una representación del $47 \%$, considerando así que las mujeres es el género predominante dentro de los encuestados.

Figura 2. Carreras profesionales

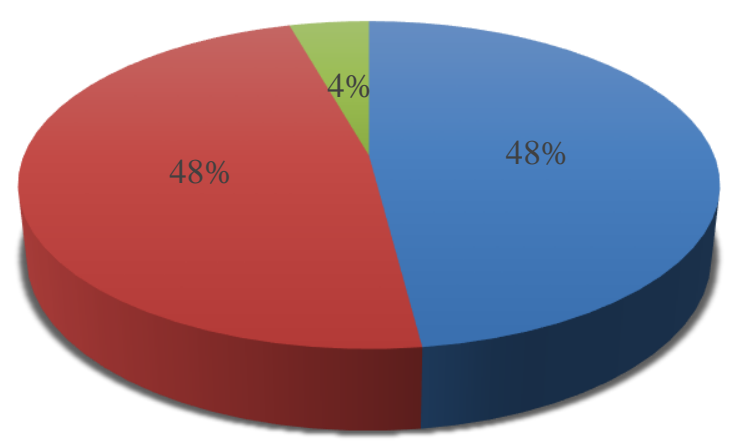

Fuente: elaboración propia.

La figura 2 expone el porcentaje de las licenciaturas que se demandan en la Facultad de Comercio y Administración Victoria, siendo la más solicitada la de Contaduría
Pública y licenciatura en Administración dentro de los encuestados representa un $48 \%$ respectivamente, seguida la de Tecnologías de la Información en un 4\%.

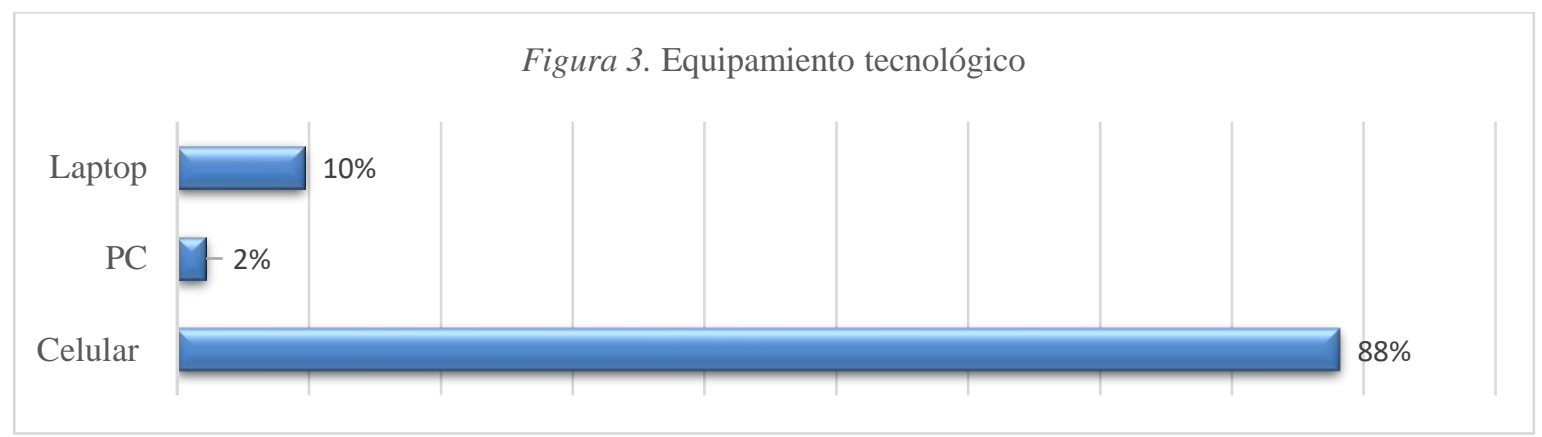

Fuente: elaboración propia.

Como se muestra en la figura 3 las proporciones de equipamiento tecnológico con lo que cuentan el alumnado, por lo que encontramos que el equipo más manipulado por los universitarios es el celular en un $88 \%$, esto alineado a los estándares nacionales e internacionales en donde la tendencia es el uso y apropiación del celular como tecnología 
de uso cotidiano, cabe hacer mención que el $10 \%$ cuenta con una laptop para atender a sus actividades académicas en modalidad en línea, y se puede considerar el desuso de la computadora de escritorio como tecnología tradicional, por lo que los usuarios buscan otras alternativas con mucho mas movilidad y practicidad como los son los dispositivos portátiles.

Figura 4. Con la implementación de las TIC, será más fácil plantear dudas o consultas.

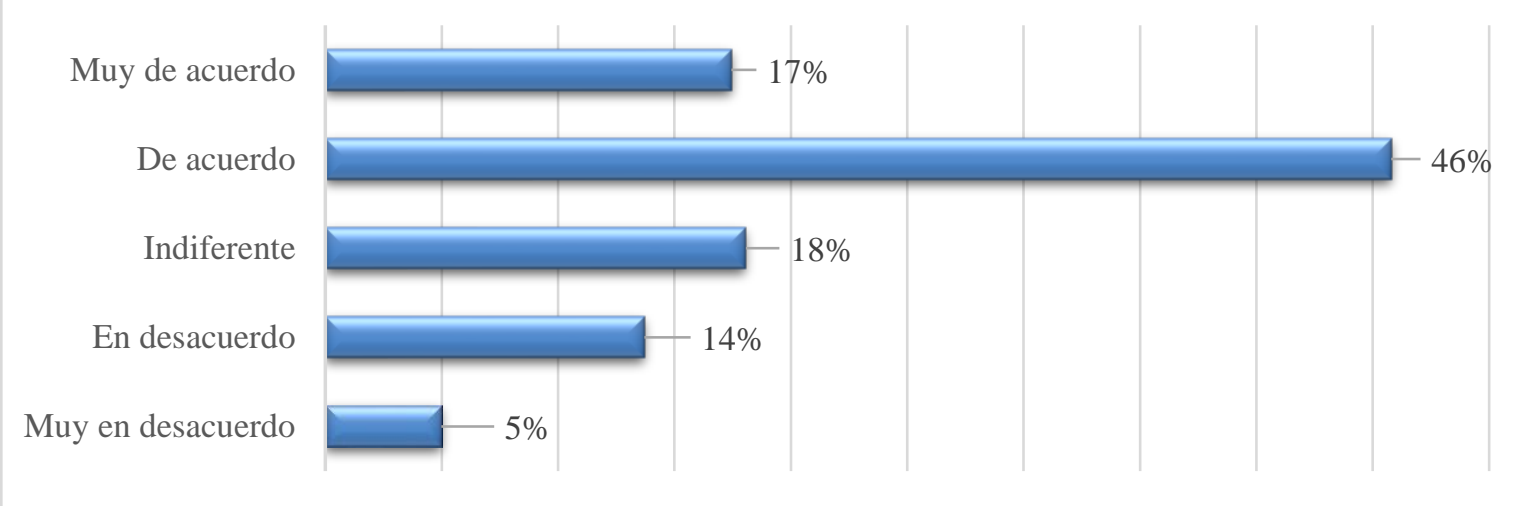

Fuente: elaboración propia.

La figura 4 nos presenta el porcentaje de apreciación de los alumnos universitarios con relación que la implementación de las TIC en sus actividades académicas, será más fácil plantear dudas o consultas, a través de los datos obtenidos en los cuestionarios aplicados en la institución educativa objeto de estudio, se observa que un contundente $63 \%$ del alumnado que curso el periodo otoño 2020 tienen la percepción de que el utilizar las Tecnologías de la Información y Comunicación facilitará enormemente la resolución de dudas o preguntas y así mejorará la calidad de los procesos de enseñanza - aprendizaje.

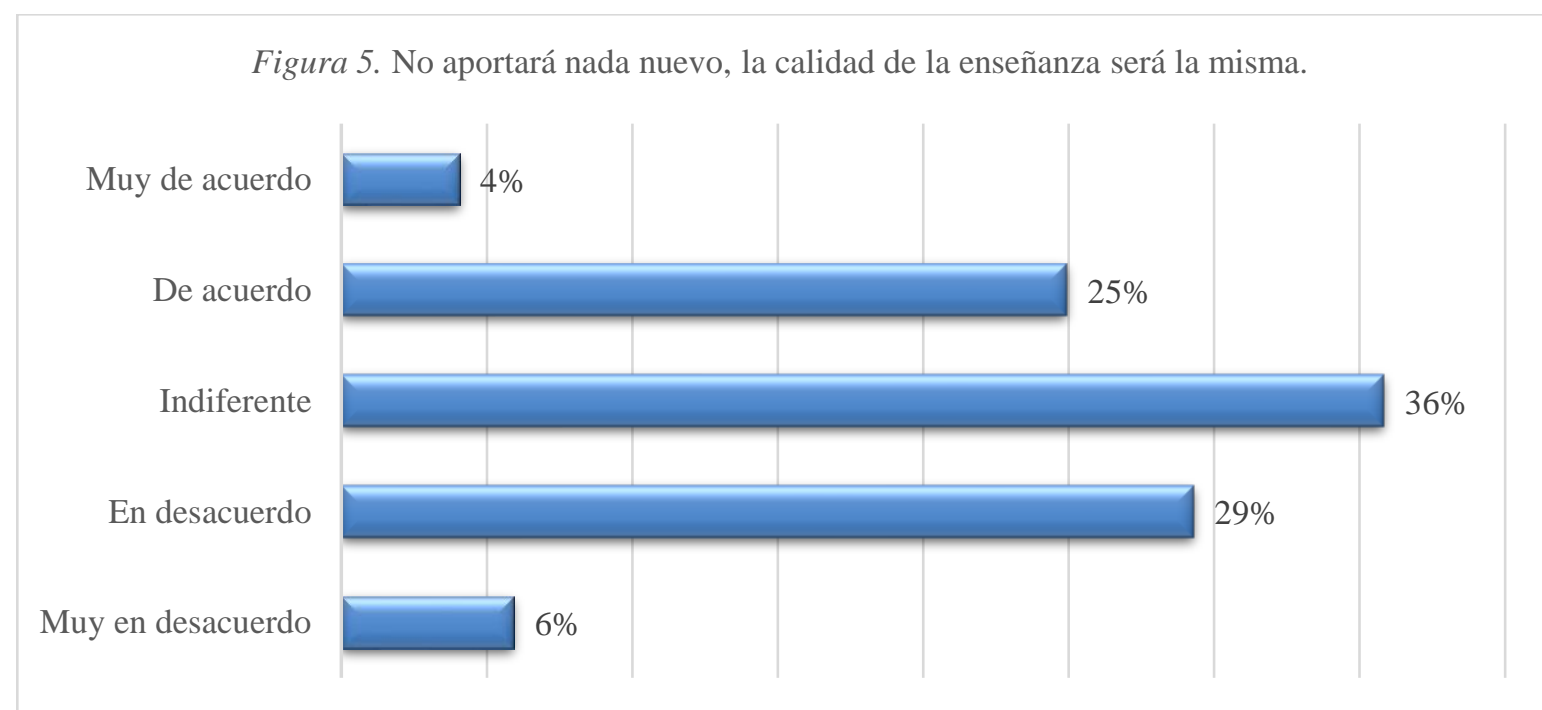

Fuente: elaboración propia.

La figura 5 revela porcentajes inquietantes con respecto a que las innovadoras herramientas tecnológicas no aportaran nada nuevo a la calidad del proceso 
de enseñanza-aprendizaje, encontrando una apreciación del $29 \%$ por parte del alumnado universitario en cuanto que dicha adopción tecnológica no ayudará a nada nuevo, contra un $35 \%$ de los encuestados que rechazan dicha afirmación, pero lo preocupante es que un $36 \%$ está indeciso, dudando si realmente es un beneficio el uso y manejo de las nuevas estrategias y que puedan contribuir en sus procesos académicos para ser frente a las exigencias de este entorno cada vez más digitalizado.

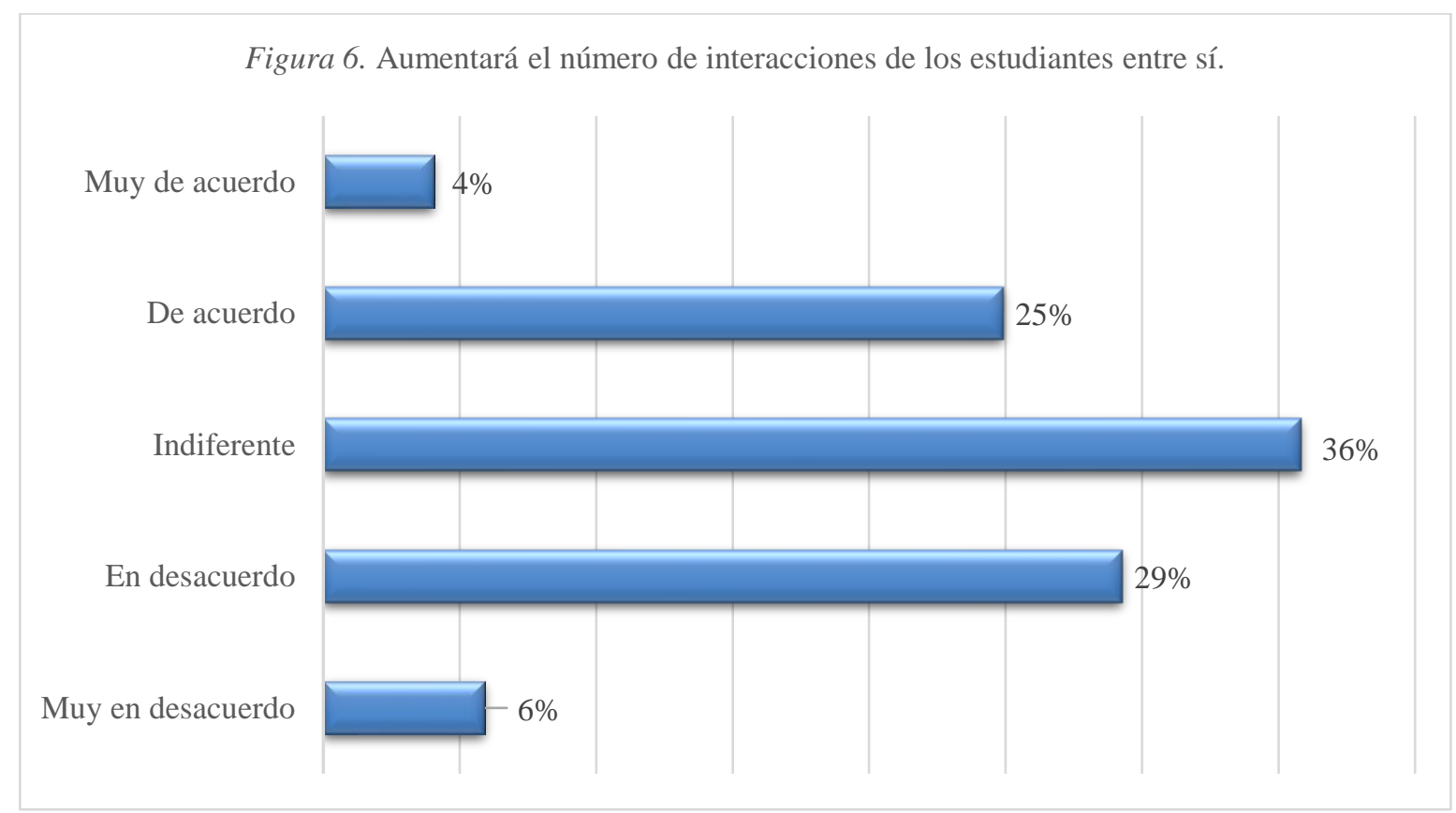

Fuente: elaboración propia.

Como se ilustra en la figura 6 , una frecuencia del $35 \%$ en donde el alumnado percibe que no aumentará las interacciones entre ellos, un $29 \%$ que si esta de acuerdo en dicho aumento y un $36 \%$ que se encuentra en una posición indiferente, en donde no se convencen de la proyección de los medios digitales a través de sus diversificadas herramientas y que el único propósito es el de construir un aprendizaje que favorezca su actividad personal y profesional.

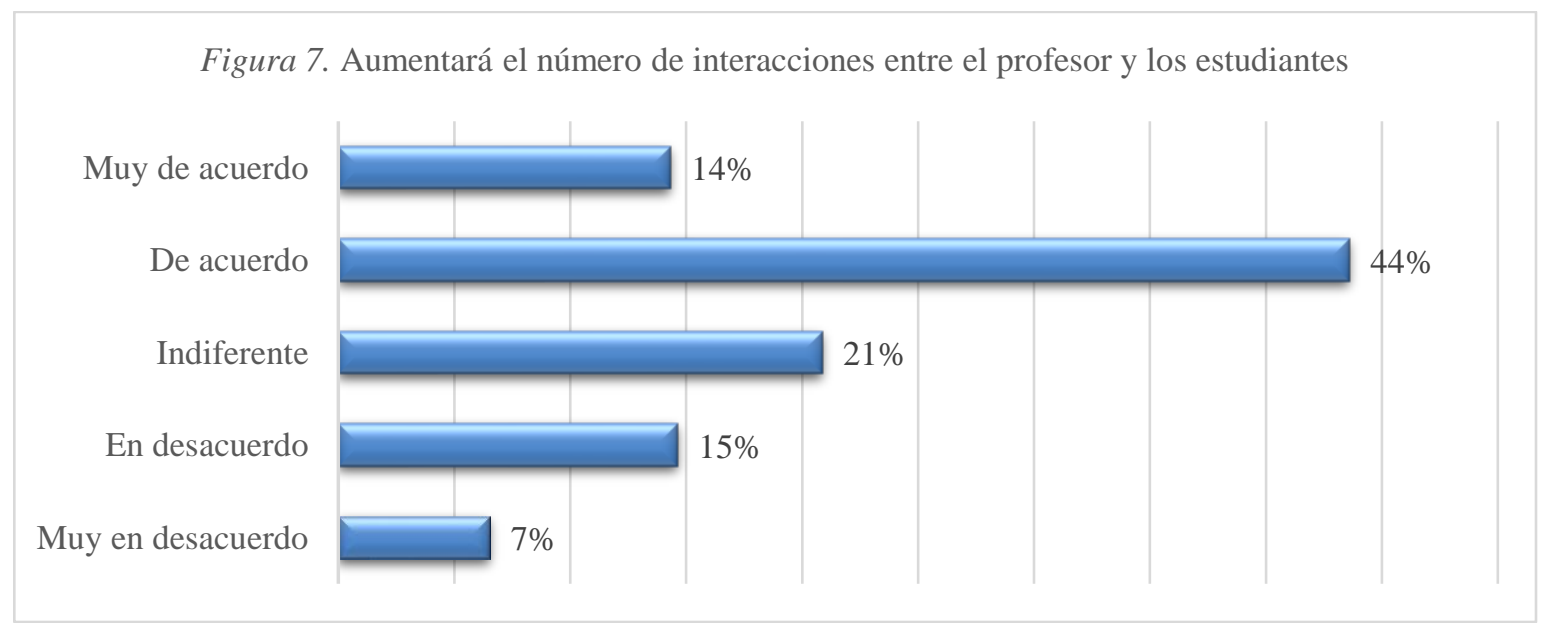

Fuente: elaboración propia. 
La figura 7 ejemplifica los resultados proporcionados por el instrumento donde un $58 \%$ del alumnado señala que aumentará el número de interacciones entre el profesor y los estudiantes, conscientes que las herramientas digitales es un vínculo que ofrece una comunicación sincrónica y asincrónica para llevar a cabo sus actividades académicas en esta nueva modalidad en línea, considerando que el uso de las TIC son instrumentos en etapas de acompañamiento en muchos de los procesos educativos, por lo que el manejo de estas pudiera ser complejo y en muchos casos estresante tanto para el alumnado y el profesorado universitario.

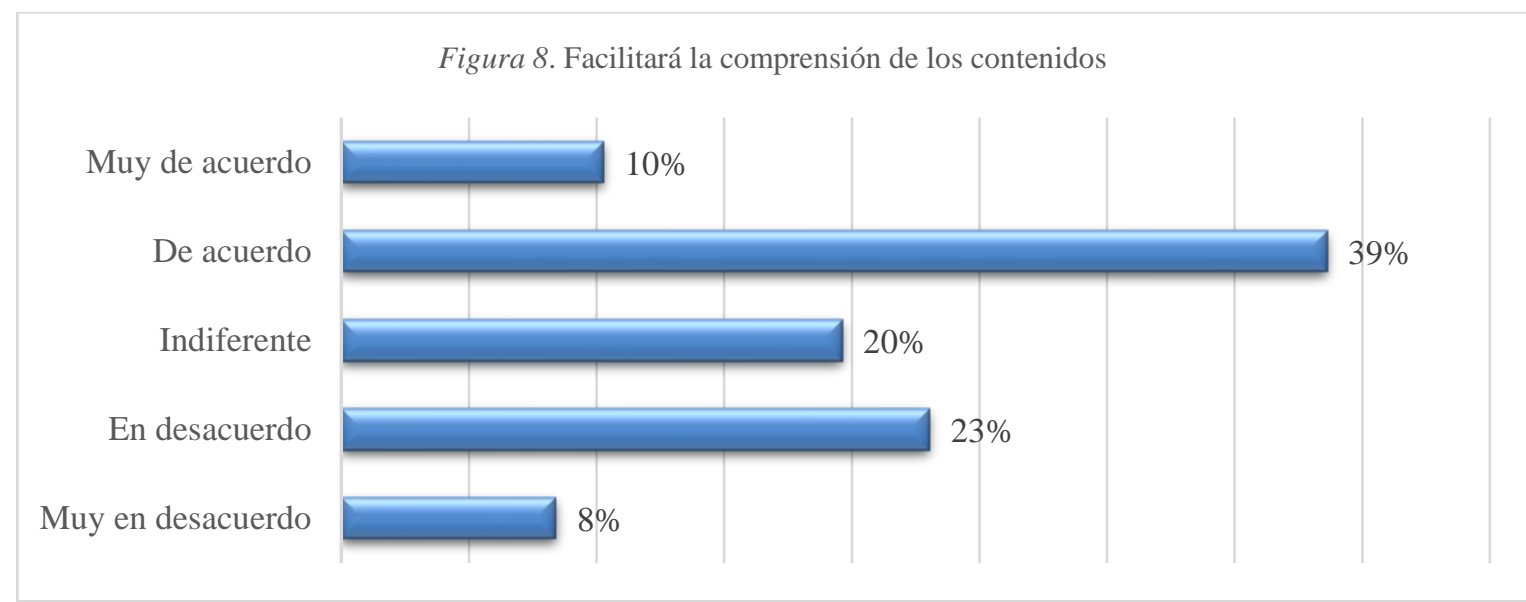

Fuente: elaboración propia.

La figura 8 proyecta que los estudiantes universitarios perciben que la utilización de medios tecnológicos y digitales facilitará la comprensión de los contenidos de las diversas asignaturas, donde un $49 \%$ está de acuerdo con dicha afirmación, por lo que el reto del docente universitario será poder lograr la inclusión por parte de sus alumnos a poder usar y manejar dichas tecnologías, aportando técnicas y estrategias innovadoras y al mismo tiempo que los alumnos puedan apropiarse de ellas, logrando así beneficiarse y alcanzar mejorar su proceso enseñanza-aprendizaje en el marco de la educación en línea.

Figura 9. No será necesario asistir a tutorías ni asesorías académicas

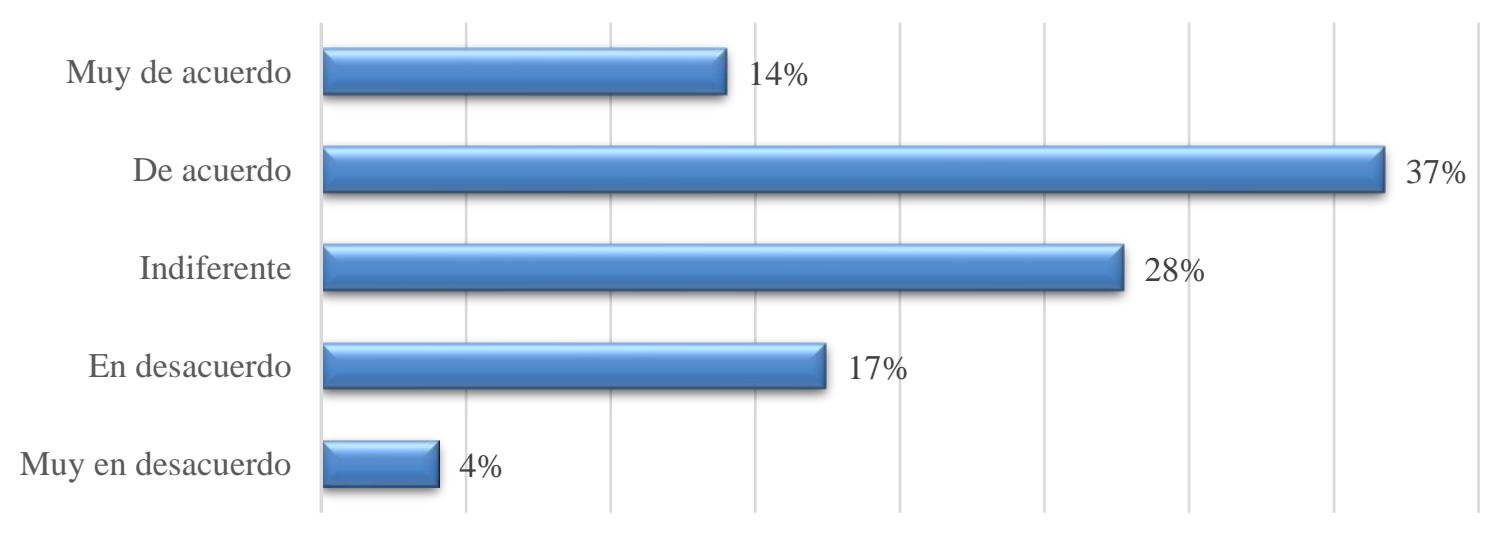

Fuente: elaboración propia.

En la siguiente figura 9 señala que los estudiantes universitarios aprecian en un $51 \%$ que no será necesario asistir a tutorías ni asesorías académicas, dado que la interconectividad que ofrecen las nuevas tecnologías suplen en cierta medida el 
acompañamiento presencial del profesor, inclusive la potencializa ya que estas herramientas nos permiten estar en permanente comunicación, siempre que los alumnos y maestros las pueden explotar y así lograr el mayor provecho posible en sus actividades extracurriculares.

Figura 10. Descenderá el número de estudiantes, con respecto a las clases presenciales

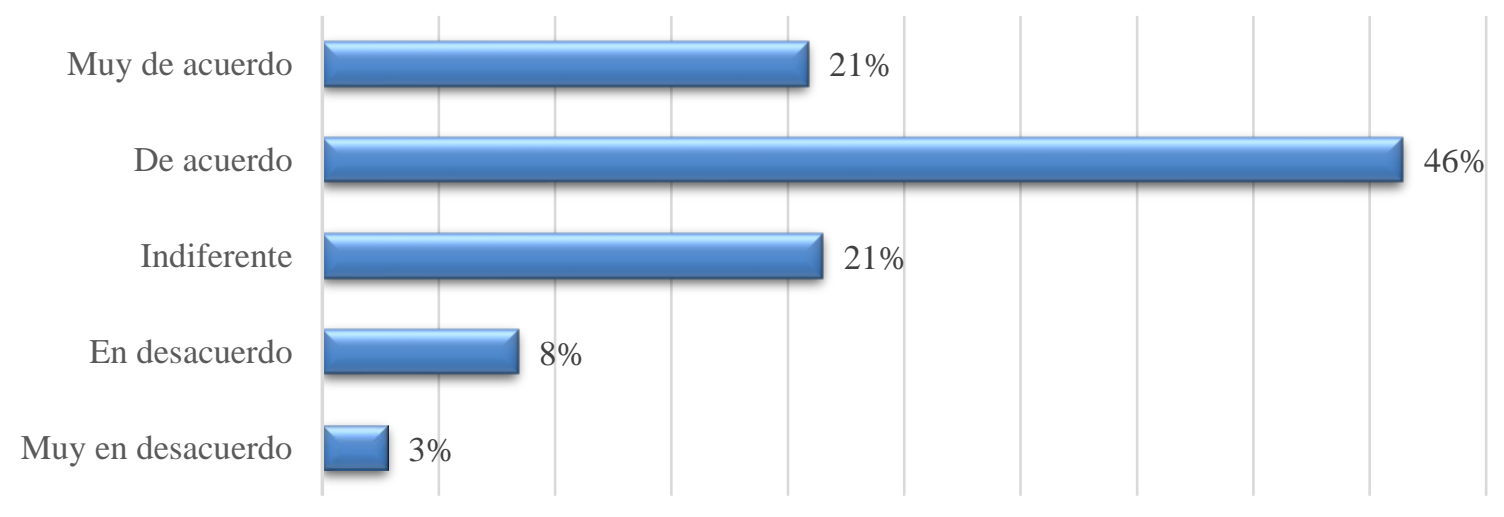

Fuente: elaboración propia.

La figura 10 muestra que los universitarios están totalmente de acuerdo en que descenderá el número de estudiantes, con respecto a las clases presenciales, ya que están conscientes que el uso de las tecnologías traerán grandes beneficios más que pérdidas de tiempo, por lo que el profesorado tendrá que sustituir las actividades tradicionales y buscar nuevas opciones que enriquezcan al alumnado universitario inmerso en un universo completamente tecnológico.

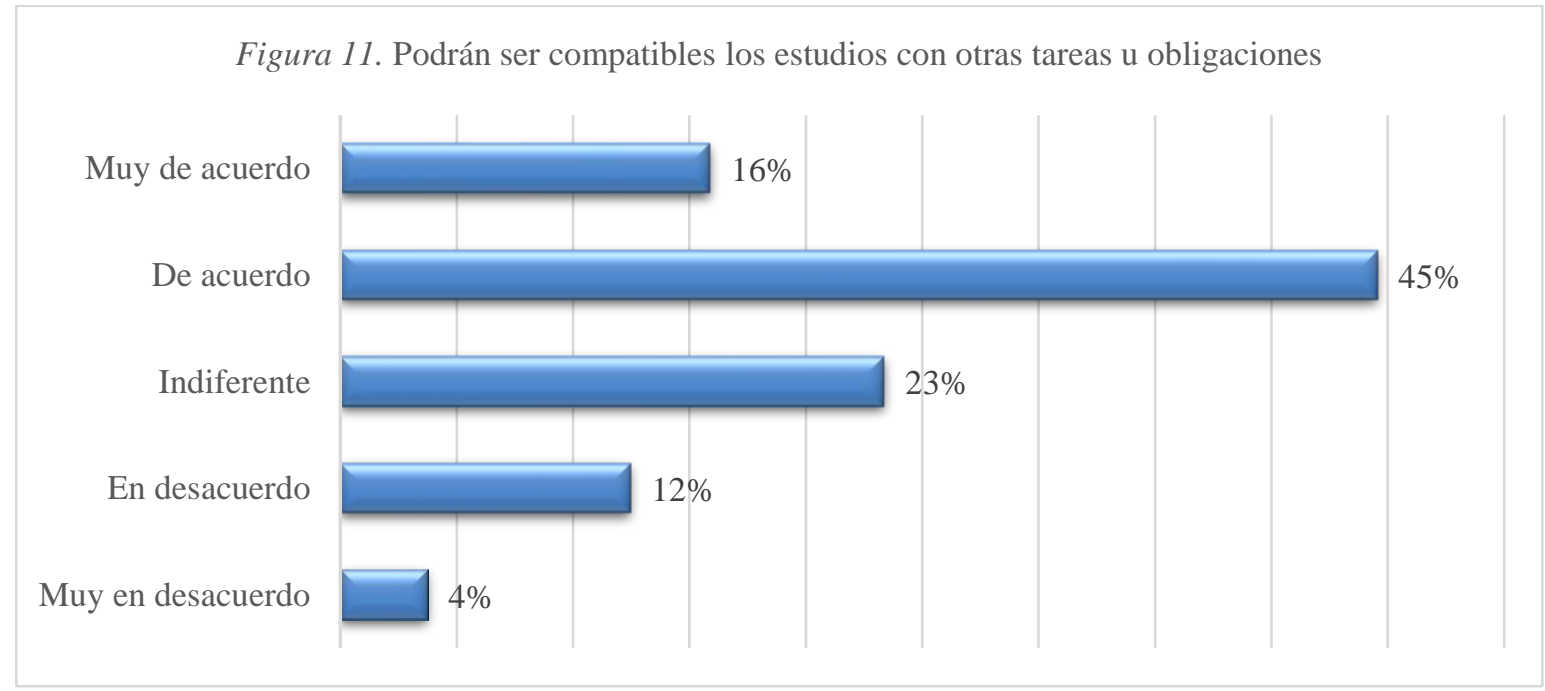

Fuente: elaboración propia.

La figura 11 gráfica que los estudiantes universitarios podrán ser compatibles sus estudios con otras tareas u obligaciones, los datos revelan que un $61 \%$ de los encuestados percibe tal aseveración, por lo que el estudiante universitario podrá alternar actividades como prácticas profesionales o servicio social sin tener problemas de horarios con sus clases en línea. 


\section{CONCLUSIONES}

De los resultados obtenidos podemos concluir que los profesores deben utilizar las TIC para mejorar la calidad de los procesos de enseñanza aprendizaje, este será un enorme desafío para el docente, en donde deberá de explorar en nuevas estrategias para que el estudiante universitario pueda ser un elemento proactivo en el desarrollo de dichos procesos, haciendo uso de las tecnologías que se encuentran a su alcance, por otro lado el rol tradicional del docente tendrá que adaptarse a los nuevos tiempos digitales por lo que el profesor habrá que convertirse en un facilitador del aprendizaje, buscando que el alumno encuentre su independencia y logre su autonomía en logro de adquirir nuevos conocimientos, de lo anterior es completamente entendible que el profesor universitario acumulará más trabajo del que venía desarrollando tradicionalmente en las aulas, en donde el solo hecho de incorporar nuevas actividades en los ambientes virtuales, implica un mejoramiento continuo en la labor docente y por ende más esfuerzo mental y más tiempo invertido.

Con respecto alumnado universitario, este señala que los ambientes virtuales pueden contribuir a aclarar dudas, a interactuar con los profesores y compañeros, por lo que el estudiante no considera una pérdida de tiempo el uso de las tecnologías, si cree que tendrá más uso social o recreativo que académico, de tal modo que el profesor tendrá que concientizar a sus alumnos de la importancia que traerá el uso de las TIC y romper con el esquema tradicional que se tiene de las tecnologías.

De lo expuesto anteriormente, no se podrá lograr un uso y apropiación de las tecnologías si nuestros alumnos no cuentan con el equipamiento necesario, la investigación arroja que cuentan con la infraestructura necesaria, pero el cumplimento de este apartado estará en función en gran medida de los indicadores socioeconómicos como son los ingresos por familia para poder adquirir dicha tecnología.

El profesor y el alumno universitarios tienen grandes retos como desafíos que deberán afrontar, ya que el contexto es muy cambiante por lo que tendrán que incorporarse a un proceso de adaptación continuo, y cada uno cumplir el rol que le corresponde, con el sentido de lograr alcanzar el proceso enseñanza aprendizaje en la nueva modalidad en línea. 


\section{REFERENCIAS}

Baker, D. (2011). Designing and orchestrating online discussions. MERLOT Journal of Online Learning and Teaching, 7(3), 401- 411. https://bit.ly/3goHYTN

Brodie, R. J., Ilic, A., Juric, B., \& Hollebeek, L. (2013). Consumer engagement in a virtual brand community: An exploratory analysis. Journal of Business Research, 66, 105-114. https://doi.org/10.1016/j. jbusres.2011.07.029

Cabero, J., \& Llorente, M. (2006). Capacidades tecnológicas de las TIC por los estudiantes. Escuela Superior de Gestión Comercial y Marketing, ESIC.

Caicedo, A., \& Rojas, T. (2014). Creencias, conocimientos y usos de las TIC de los profesores universitarios. Educ.Educ., 17(3), 517-533.

Castaño-Muñoz, J., Duart, J., \& Vinuesa, T. (2014). The internet in face-to-face higher education: Can interactive learning improve academic achievement? British Journal of Educational Technology 45(1), 149-159. En: https://doi.org/10.1111/ bjet.12007

Chakraborty, M., \& Nafukho, F. M. (2015). Strategies for virtual learning environments: Focusing on teaching presence and teaching immediacy. Internet Learning, 4(1). En: https://doi.org/10.18278/ il.4.1.

Coll, C. (2004). Psicología de la educación y prácticas educativas mediadas por las tecnologías de la información y la comunicación. Una mirada constructivista. Revista Electrónica Sinéctica, (25), 1-24.

Cuban, L. (2001). Oversold and underused: computers in the classroom Cambrige, Massachusetts, London. Harvard University.

Del Moral, M., Villalustre, L., \& Neira, M. (2014). Oportunidades de las TIC para la innovación educativa en las escuelas rurales de Asturias. Aula Abierta (47), 61-67.

Ferro, C., Martínez, A., \& Otero, M. (2009). Ventajas del uso de las TIC en el proceso de enseñanzaaprendizaje desde la óptica de los docentes universitarios españoles. EDUTEC, Revista Electrónica de Tecnología Educativa.

García, N., Rivero, M., \& Ricis, J. (2020). Brecha Digital en tiempo del COVID-19. Revista Educativa Hekademos, 28.

Gómez, G. (2008). El uso de la tecnología de la información y la comunicación y el diseño curricular. Educación, 32(1), 77-97.

Gómez, L., \& Macedo, J. (2010). Importancia de las TIC en la educación básica regular. Investigación Educativa, 14(25), 209-224.

Kiridis, A., Drossos, V., \& Tsakiridou, H. (2006). Teachers facing information and communication technology (ICT): the case of Greece. Journal of Technology and Teacher Education.

Mason, R. (1998). Models of online courses. ALN Magazine, 2(2).

Moallem, M. (2015). The impact of synchronous and asynchronous communication tools on learner selfregulation, social presence, immediacy, intimacy, and satisfaction in collaborative online learning. The Online Journal of Distance Education and e-learning, 3(3), 55-77. En: https://bit. ly/3aK571

Morán, L., \& Myringer, B. (1999). Flexible learning and university change. Higher Education Through Open and Distance Learning, 57-72.

Ragusa, A. T. (2017). Technologically mediated communication: student expectations and experiences in a FOMO society. International Journal of Educational Technology in Higher Education, 14, 39. En: https://doi. org/10.1186/s41239-017-0077-7

Sáez, J. (2010). Utilización de las TIC en el proceso de enseñanza aprendizaje, valorando la 
incidencia real de las tecnologías en la práctica docente. Revista Docencia e Investigación. Salinas, J. (1997). Enseñanza flexible, aprendizaje abierto. Las redes como herramientas para la formación. Málaga: ICE / Universidad de Málaga.

Salinas, J. (1999). El rol del profesorado universitario ante los cambios de la era digital. Actas del I Encuentro Iberoamericano de Perfeccionamiento Integral del Profesor Universitario. Caracas: Universidad Central de Venezuela.

Sanabria, A., \& Hernández, C. (2011). Percepción de los estudiantes y profesores sobre el uso de las tic en los procesos de cambio e innovación en la enseñanza superior. Aloma (29), 273 290.

Sánchez, L., Pardo, M., \& Izquierdo, J. (2010). La dinámica del proceso de formación para la investigación científica en la educación superior sustentada en las tecnologías de la información y las comunicaciones. Pedagogía Universitaria, 15(2), 67-81.

UNESCO. (1997). UNESCO para la Educación en el contexto del seguimiento de la Quinta Conferencia Internacional de Educación de las Personas Adultas (CONFINTEA V), llevada a cabo en Hamburgo en el año de 1997. Nuevas tecnologías de la información y educación de adultos. 